\title{
Conjectures sur le social et les sciences sociales
}

\section{Dany-Robert Dufour}

\section{OpenEdition \\ Journals}

Édition électronique

URL : http://journals.openedition.org/ress/506

DOI : $10.4000 /$ ress.506

ISSN : 1663-4446

\section{Éditeur}

Librairie Droz

\section{Édition imprimée}

Date de publication : 1 décembre 2003

Pagination : $51-62$

ISBN : 2-600-00912-4

ISSN : 0048-8046

Référence électronique

Dany-Robert Dufour, "Conjectures sur le social et les sciences sociales », Revue européenne des sciences sociales [En ligne], XLI-127 | 2003, mis en ligne le 30 novembre 2009, consulté le 04 mai 2019. URL : http://journals.openedition.org/ress/506 ; DOI : 10.4000/ress.506 
Dany-Robert DUFOUR

\section{CONJECTURES SUR LE SOCIAL ET LES SCIENCES SOCIALES}

\section{LA LOGIQUE DE LA MARCHANDISE, LE SOCIAL ET L'ÉTAT}

Lorsqu'on parle de science sociale, on suppose au moins une chose: que le social préexiste au discours que l'on tient sur lui. Or, j'avoue ne plus bien savoir comment situer le social dans des sociétés devenues entièrement marchandes comme les nôtres. On sait en effet que plus l'échange de marchandises se généralise, plus il implique la disparition des anciens rapports sociaux (qu'ils soient fondés sur l'échange symbolique, sur des rapports communautaires ou sur des rapports d'autorité). Et l'on sait également que cette disparition n'est pas compensée par l'instauration de nouveaux rapports sociaux. Ou, du moins, s'ils apparaissent, ces rapports sont entièrement déductibles des rapports économiques noués entre ceux qui échangent des marchandises - j'entends bien sûr, au titre des marchandises offertes sur le marché, la force de travail. Bref, dans le capitalisme, les rapports sociaux sont essentiellement réductibles à des rapports économiques.

Dans les sociétés marchandes en effet, l'échange des produits est ce qui relie les hommes et cet échange n'est pas médiatisé par un rapport social organique, mais par le simple auto-déploiement du mouvement de la valeur. C'est la valeur qui, sous la forme visible de l'argent, donne alors forme à l'organisation sociale. La sociologie marxienne est incontournable sur ce point. Marx note ainsi dans les Grundrisse I que «l'argent est la communauté et ne peut en tolérer aucune autre qui lui soit supérieure $»^{1}$. L'argent étant devenu ce qui fait lien social, il ne peut que détruire les communautés organiques en instituant à leur place des communautés abstraites ayant gommé les qualités concrètes de ses membres.

C'est pourquoi, pour compenser cette « disparition» du social, le politique est indispensable. Ce que le philosophe Anselm Jappe résume ainsi dans son dernier ouvrage:

la logique de la valeur se base sur des producteurs privés qui n'ont pas de lien social entre eux, et c'est pourquoi elle doit produire une instance séparée qui s'occupe de l'aspect général. L'Etat moderne est donc créé par la logique de la marchandise ${ }^{2}$.

J'agrée à ce propos, quoique la dernière phrase me semble un peu rapide: à vrai dire, ce n'est pas la logique de la marchandise qui crée (directement) le politique.

Karl Marx, Manuscrits de 1857-1858 (Grundrisse I), Éd. J.-P. Lefebvre, Éditions sociales, Paris, 1980, p. 161.

2 Anselm Jappe, Les aventures de la marchandise, Denoël, Paris, 2003, p. 167. 
Cette création est en effet indirecte: c'est la destruction des anciens rapports sociaux, opérée par la logique de la marchandise, qui implique de compenser cette disparition. Le politique n'est en somme pas pensable autrement que comme ce qui supplée à la disparition des anciens rapports sociaux provoquée par le règne de la valeur et de la marchandise. Et, de fait, là où il existe de véritables rapports sociaux puissants, le politique n'existe pas comme une instance spécifique qu'on se souvienne, par exemple, des célèbres analyses de Clastres sur les sociétés sans Etat. Ceci est d'ailleurs congruent avec l'apparition, dans les grandes cités marchandes de la Renaissance, du politique comme instance spécifique repérée comme telle par Machiavel. Lorsque Claude Lefort parle du politique comme forme symbolique, il faut bien comprendre ce que cela signifie: le symbolique qui, dans les formes antérieures au capitalisme, était dans le social, est entièrement passé dans l'instance politique.

On pourrait dire, pour l'instant et pour simplifier, que l'économique réfère aux intérêts privés cependant que le politique réfère à l'intérêt universel. Rien n'est bien sûr plus délicat que de définir des intérêts universels et l'on sait le sort que les «théoriciens du soupçon» du XIX ${ }^{\mathrm{e}}$ siècle ont fait subir aux principes universels que les Lumières du siècle précédent s'étaient employés à établir. Ils ont en effet dénoncé l'intérêt universel et ses principes «moraux» comme spécieux: ils ne représentaient pour Marx que l'habillage de la domination exercée sur les masses par les exploitants ou, à l'inverse, que la tyrannie exercée par les faibles sur les forts pour Nietzsche, ou encore que le rôle de censure exercée par le surmoi au profit de la civilisation pour Freud. Mais, qu'ils soient dénonçables de plusieurs façons, n'a jamais empêché le politique de devoir reposer sur un certain nombre de principes transcendantaux (religieux, moraux, idéaux...).

On peut donc dire que le social, dans les sociétés marchandes modernes, était entièrement tenu par le politique. En d'autres termes, c'est le politique qui était en charge de maintenir la société dans tous états, comme vivable pour ses membres. Outre une politique économique, il devait ainsi y avoir une politique de la famille, une politique des associations, une politique de la culture, une politique de la nation et de ses composantes, etc. Il est d'ailleurs arrivé dans la période moderne des moments où le politique a été considérablement investi, notamment le mouvement ouvrier, jusqu'au délire même - le cas de l'Union soviétique et de sa proliférante bureaucratie voulant tout réguler et tout contrôler au nom du Peuple suffira à illustrer cette volonté de domination absolue sur la société civile. Mais, si l'on ne va pas jusqu'aux extrêmes, on peut dire que, dans la modernité, les intérêts privés économiques étaient (plus ou moins bien) contrôlés par le politique au nom de l'intérêt universel. Et que le social, tenu à bout de bras par le politique, y trouvait (plus ou moins bien) son compte.

Or, nous arrivons à une époque nouvelle qui est en train de promouvoir la liquidation méthodique du politique. C'est l'époque connue sous le nom de néolibérale.

\section{LA LOGIQUE DE LA MARCHANDISE CONTRE L'ÉTAT}

Il est apparu aux économistes (en premier lieu ceux de l'école de Chicago) que le politique constituait un frein à la circulation sans cesse élargie des marchan- 
dises. D'une certaine façon, ils ont raison: le politique est bien le contre-pouvoir essentiel à la constitution d'un monde entièrement fondé sur l'économique et la circulation des marchandises. Mais ces économistes ont simplement voulu oublier qu'il y avait de bonnes raisons à cette limitation. Or, l'étrange est qu'ils aient réussi à convaincre les stratèges politiques que le politique devait cesser de jouer son rôle. Ce hara-kiri du politique restera probablement l'un des grands mystères de la fin du $\mathrm{XX}^{\mathrm{e}}$ siècle à élucider - mais c'est là est une autre histoire.

Cette défaillance du politique ne peut bien sûr qu'entraîner de considérables effets délétères sur le social en tant qu'il était jusqu'alors entièrement tenu par le politique. Qu'on dissolve dans ces conditions le politique et le social s'effondre. Il convient toutefois de remarquer que ces effets sur le social sont la conséquence d'une attaque en règle comme les principes transcendantaux sur lesquelles le politique reposait.

La gouvernance néo-libérale actuelle est en effet construite sur l'idée qu'à un minimum de gouvernement politique correspond un maximum de rendement économique. Comme il y avait une servitude volontaire dans l'Ancien régime, il existe désormais un sacrifice volontaire du politique. De fait, le pouvoir actuel ne «veut» plus rien, rien que la meilleure adaptation possible à une conjoncture et à une évolution qui le dépassent. La «modernisation» (des entreprises, de l'école, des institutions $\left.{ }^{3} . ..\right)$ se présente ainsi comme un gigantesque tropisme à l'échelle planétaire, une sorte de loi naturelle, une poussée sourde et irrépressible de l'évolution. Ce serait la «force des choses» qui exige soumission et adaptation vitales et non les détenteurs d'un pouvoir devenu flou, mou, secondaire et gestionnaire. L'absence d'un véritable gouvernement, c'est-à-dire d'une institution dont la légitimité est nécessairement extérieure aux intérêts économiques, abolit ce que Hannah Arendt appelait l'autorité tout en rendant la puissance occulte. L'affaiblissement de l'Etat n'annonce pas, loin s'en faut, celui de la domination socioéconomique, mais le passage à une nouvelle forme de domination, sournoise et maligne, par laquelle le pouvoir véritable devient anonyme, informe et non localisable: «nous sommes devant une tyrannie sans tyran» déclarait à cet égard Hannah Arendt dans ses études prémonitoires sur les Etats-Unis ${ }^{4}$.

Signe de cette disparition de l'autorité: nous assistons ouvertement à la promotion de l'anomie, à la levée des interdits et de tout ce qui peut en imposer à la pure impétuosité des appétits. S'il est en effet un impératif post-moderne, c'est celui de la transgression des interdits - voilà pourquoi cette tyrannie sans tyran diffuse un parfum libertaire fondé sur la proclamation de l'autonomie de chacun et sur «l'extension indéfinie de la tolérance dans tous les domaines $»^{5}$.

L'écrasement de la citoyenneté sur la société civile marchande, seulement constituée de l'ensemble conflictuel des intérêts particuliers, rend impossible la nécessaire dialectique entre le corps social et sa représentation politique. La notion d'Etat, telle qu'elle fonctionne depuis les Lumières, tombe ainsi en désuétude. A la limite, il n'y a plus de chose publique, mais seulement des intérêts

Voir sur ce point, Jean-Pierre Le Goff, La Barbarie douce, la modernisation aveugle des entreprises et de l'école, La Découverte, Paris, 1999.

${ }^{4} \quad$ Hannah Arendt, Du mensonge à la violence, Calmann-Lévy, Paris, 1972, p. 181.

$5 \quad$ Pierre-André Taguieff, Résister au bougisme, Mille et une nuits, Paris, 2001, p. 15. 
privés. Or, comme le disait Rousseau dans Le Contrat Social (Livre III, chapitre IV):

Rien n'est plus dangereux que l'influence des intérêts privés dans les affaires publiques.

L'humanité se trouve réduite à une collection d'individus calculateurs mus par leurs seuls intérêts rationnels, en concurrence sauvage les uns avec les autres l'utilitarisme de Adam Smith l'ayant emporté (pour longtemps semble-t-il) sur l'injonction morale des Lumières. En définitive, cette anthropologie, dont le célèbre mot d'ordre «laisser-faire» avouait par avance l'absence de principe, ouvre un nouvel espace sociétal, complètement épuré, prosaïque, trivial, nihiliste, empreint d'un nouvel et puissant darwinisme social où la valeur, désormais unique, passe d'une main à l'autre sans autre forme de procès et quelles qu'en soient les modalités: les «plus adaptés » peuvent légitimement profiter de toutes les situations cependant que les «moins adaptés» sont tout simplement abandonnés, voire appelés à disparaître. C'est là une profonde remise en cause de la civilisation puisque se trouve abandonné le traditionnel devoir «biopolitique» (Foucault, Agamben) incombant à tout Etat de protection de ses populations.

Cet étiolement de l'Etat est une conséquence directe du pragmatisme, de l'utilitarisme et du «réalisme» contemporains qui entendent «dégraisser»les échanges fonctionnels de la surcharge symbolique et politique qui les équilibraient et les rendaient possible comme la quille d'un navire. Le moins d'Etat correspond à un affaiblissement considérable des valeurs qui régulaient l'échange humain. Ces «valeurs» postulées relevaient d'une culture (dépositaire de principes moraux, de canons esthétiques, de modèles de vérité) et comme telles, elles pouvaient différer d'autres valeurs, soit s'y opposer. Or, la disparition de ces valeurs (pour lesquelles on pouvait mourir) rend bien sûr désuète toute forme de luttes entre valeurs concurrentes - ce qui se manifeste par la désuétude contemporaine du débat politique, devenu le comble de l'ennui pour la plupart des contemporains.

L'Etat ne pèse plus presque plus rien devant cet idéal de fluidité, de transparence, de circulation et de renouvellement qui ne peut s'accommoder du poids historique de ces valeurs culturelles. En ce sens, l'adjectif «libéral» désigne la condition d'un homme «libéré» de toute attache à des valeurs. Tout ce qui se rapporte à la sphère transcendante des principes et des idéaux, n'étant pas convertible en marchandises ou en services, se voit désormais discrédité. Les valeurs (morales) n'ont pas de valeur (marchande). Ne valant rien, leur survie ne se justifie plus dans un univers devenu intégralement marchand ${ }^{6}$.

De fait, ces mutations sont contemporaines d'une extension sans précédent du modèle de l'échange marchand. Cette mort programmée du politique est en rapport direct avec la mutation que l'on observe depuis une bonne vingtaine d'années dans le capitalisme. Le néo-libéralisme, comme on nomme sommairement ce nouvel état du capitalisme, est en train de défaire toutes les formes d'échanges qui subsistaient par référence à un garant absolu ou méta-social des échanges. Pour aller à la fois vite et à l'essentiel, on pourrait dire qu'il fallait un étalon - l'or par

Sur ces questions, cf. Patrick Berthier, Dany-Robert Dufour, «Vers un nouveau nihilisme», in Le Débat, janvier 2003, Gallimard, Paris. 
exemple - pour garantir les échanges monétaires ${ }^{7}$, comme il fallait un garant symbolique (la Raison, par exemple) pour permettre les discours philosophiques, comme il fallait un Etat pour éviter la «guerre de tous contre tous» (Hobbes). Or, on cesse désormais de se référer à toute valeur transcendantale pour se livrer aux échanges. Comme le dit Marcel Gauchet, nous avons désormais affaire à «des acteurs qui se veulent rigoureusement déliés et sans rien au-dessus d'eux pour empêcher la maximisation de leurs entreprises ${ }^{8}$. Les échanges ne valent plus en tant que garantis par une puissance supérieure (d'ordre transcendantale ou morale), mais par ce qu'ils mettent directement en rapport en tant que marchandises.

C'est le poids du symbolique dans les échanges humains, qui a fait les beaux jours de la grande anthropologie du $\mathrm{XX}^{\mathrm{e}}$ siècle (de Mauss à Lévi-Strauss, et jusqu'à Lacan), qui se trouve de la sorte mis en cause. Si, comme le dit encore Marcel Gauchet, «la sphère d'application du modèle [de marché] est destiné à s'élargir bien au-delà du domaine de l'échange marchand $»^{9}$, alors il y a un prix à payer pour cette extension: l'affaiblissement et même l'altération de la fonction symbolique.

Ce changement radical dans le jeu des échanges entraîne en fait à une véritable mutation anthropologique. Dès lors que tout garant symbolique des échanges entre les hommes tend à disparaître, c'est la condition humaine elle-même qui change. En effet, notre être-au-monde ne peut plus être le même dès lors que l'enjeu d'une vie humaine ne tient plus à la recherche de l'accord avec ces valeurs symboliques transcendantales jouant le rôle de garants, mais est liée à la capacité de s'accorder aux flux toujours mouvants de la circulation de la marchandise. En un mot, ce n'est plus le même sujet qui est requis ici et là ${ }^{10}$. Nous commençons de la sorte à découvrir que le néo-libéralisme, comme toutes les idéologies précédentes qui se sont déchaînées au cours du $\mathrm{XX}^{\mathrm{e}}$ siècle (le communisme, le nazisme...), ne veut rien d'autre que la fabrication d'un homme nouveau. Cette nouvelle tentative est cependant particulièrement retorse en ce sens qu'elle se donne sous le jour d'une libération totale de l'individu et d'un affranchissement de toute idéologie. Elle est en fait elle-même une idéologie et sa grande force par rapport aux précédentes tient à ce qu'elle n'a pas commencé par viser l'homme lui-même au moyen de programmes de rééducation et de coercition. Elle s'est contentée d'introduire un nouveau statut de l'objet, défini comme simple marchandise, en attendant que le reste s'ensuive: que les hommes se transforment lors de leur adaptation à la marchandise, promue dès lors comme seul réel ${ }^{11}$. Le

Cf. Jean-Joseph Goux, Frivolité de la valeur, essai sur l'imaginaire du capitalisme, Blusson, Paris, 2001.

s Cf. Marcel Gauchet, La démocratie contre elle-même, Gallimard, Paris, 2002, Avant-propos, p. XXV.

$9 \quad$ Ibid, p. XXV.

10 J'examine dans Dany-Robert Dufour, L'art de réduire les têtes à l'ère du capitalisme total (Denoël, Paris, 2003) les conditions de la destruction du triple sujet de la modernité, le sujet critique (kantien), le sujet névrotique (freudien) et le sujet producteur (marxien) et de la mise en place d'un nouveau sujet, dit «post-moderne».

${ }^{11}$ Il n'y a cependant pas une once de réel de plus dans le fait d'aller au supermarché acheter un produit dont on n'a nul besoin que dans le fait d'aller invoquer un totem... 
nouveau dressage de l'individu s'effectue donc au nom d'un «réel » à quoi il vaut mieux consentir que s'opposer: il doit toujours paraître doux, voulu, désiré, comme s'il s'agissait d'une série d'entertainments (exemples: la télévision, la pub... qui, en jouant le rôle d'alternatives à l'éducation, vident l'école de toute substance et la mènent tout droit à la vertigineuse crise que nous connaissons).

De façon générale, toute figure transcendante qui venait fonder la valeur est désormais récusée, il n'y a plus que des marchandises qui s'échangent à leur stricte valeur marchande. Les hommes sont aujourd'hui priés de se débarrasser de toutes ces surcharges symboliques qui garantissaient leurs échanges. La valeur symbolique est ainsi démantelée au profit de la simple et neutre valeur monétaire de la marchandise de sorte que plus rien d'autre, aucune autre considération (morale, traditionnelle, transcendante, transcendantale...) ne puisse faire entrave à sa libre circulation. Il en résulte une désymbolisation du monde. Les hommes ne doivent plus s'accorder aux valeurs symboliques transcendantes, ils doivent simplement se plier au jeu de la circulation infinie et élargie de la marchandise.

En somme, tout est devenu monnayable. Nous sommes sortis de l'exception dont se fondait tout le reste des échanges. Exception dont rend parfaitement compte cette proposition de Kant exposée dans les Fondements de la métaphysique des mœurs [1785]:

Tout a ou bien un prix, ou bien une dignité. On peut remplacer ce qui a un prix par son équivalent; en revanche, ce qui n'a pas de prix, et donc pas d'équivalent, c'est ce qui possède une dignité ${ }^{12}$.

On ne peut le dire plus clairement: la dignité ne peut être remplacée, elle «n'a pas de prix » et «pas d'équivalent», elle réfère seulement à ce que Kant appelait l'autonomie de la volonté, c'est-à-dire cette morale moderne, non plus statique, mais dynamique. C'est pourquoi l'échange marchand ne veut rien savoir du sujet critique, c'est même tout le contraire qui est requis dans le démarchage, le marketing et la promotion (volontiers mensongère) de la marchandise. On voudrait nous assurer qu'il s'agit, dans cette récusation du sujet critique, d'un grand retour de l'utilitarisme et de la revanche tardive de Hume sur Kant. Mais comment ne pas remarquer qu'il s'agit d'un utilitarisme doublement édulcoré: d'une part, il prône la recherche du bonheur individuel beaucoup plus que la recherche du bonheur du plus grand nombre; d'autre part, il réduit et circonscrit le bonheur individuel à la seule dimension de l'appropriation de l'objet marchand.

\section{LA LOGIQUE DE LA MARCHANDISE CONTRE LE SOCIAL}

J'ai fait semblant, tout à l'heure, de considérer que le social, à l'ère moderne de la généralisation de l'échange marchand, était entièrement tenu par le politique. En fait, ce n'est pas tout à fait vrai. On commence à savoir qu'il est des secteurs où des formes anciennes de lien social ont survécu sans tout devoir au politique. Je fais référence ici à plusieurs recherches contemporaines, dont celle

12 Emmanuel Kant, Fondements de la métaphysique des mœurs [1785], Garnier-Flammarion, Paris, 1994, p. 116. 
du sémiologue François Flahault. Cet auteur a montré qu'il était logiquement impossible que les sociétés humaines reposent sur la seule économie marchande car cette économie ne peut tenir sans être doublée de ce qu'il appelle une «économie des personnes». En effet, pour que des biens circulent entre des personnes, il faut auparavant que celles-ci aient été instituées. Bref pour qu'il y ait des biens qui circulent entre les personnes, il faut qu'il ait des personnes formées comme telles. Ce qui les forme, c'est la culture qui constitue un milieu tiers, un monde commun qui est celui des échanges discursifs fondateurs de subjectivités. Ces échanges mettent notamment en jeu une transmission générationnelle de sorte que «la circulation de biens entre contemporains présuppose le don qu'une génération fait à la suivante ${ }^{13}$. Ce don est d'abord celui d'une langue dans laquelle se forment les nouveaux venus. Le propre de cette dette est qu'elle est impayable. En effet, elle ne réfère pas à une dette d'avoir (de celles dont on peut se libérer en payant). Elle réfère à une dette d'être. Dette proprement symbolique que le débiteur n'est pas tenu de régler, mais qu'il lui faut impérativement reconnaître au cours de sa constitution subjective. On entre là dans le cadre d'une économie symbolique et psychique.

La grande question étant aujourd'hui de savoir si cette économie des personnes n'est pas en train d'être mise au pas par l'économie marchande. Ce qui aurait bien sûr des effets considérables sur les formes mêmes de la subjectivation. J'ai tenté ailleurs d'analyser les effets de cet alignement forcé de l'économie des personnes sur l'économie des biens et je me contenterais ici d'y renvoyer ${ }^{14}$.

Jean-Claude Michéa a, de son côté, mis en lumière tout un pan de socialité qui ne doit rien à l'Etat et qu'il a regroupé sous le nom (emprunté à Orwell) de common decency ${ }^{15}$. La common decency renvoie au rôle joué par la «confiance réciproque» des membres les uns envers les autres dans la constitution des communautés ouvrières et populaires du XIX $\mathrm{X}^{\mathrm{e}}$ siècle. Michéa retient, à partir de notes éparses d'Orwell, les traits suivants. La common decency réfère à un sentiment intuitif des choses qui ne doivent pas se faire dès lors qu'on cherche à maintenir les conditions d'une existence quotidienne commune. La common decency emprunte une partie de ses traits à la tradition chrétienne et à l'héritage de la Révolution française, mais elle n'en possède pas moins une réelle universalité. Elle peut en effet s'autoriser de fondations métaphysiques diverses allant des codes traditionnels jusqu'à des formes de conscience morale plus personnelles. Les codes de la common decency sont philosophiquement et psychologiquement accessibles à l'ensemble des classes de la société. La common decency renvoie à un champ de relations vastes qui va de l'entraide bienveillante à la grande solidarité en passant par la simple politesse. Les codes de la common decency mettent à l'index l'«indécence» qui pousse à s'élever au-dessus de ses semblables ou à s'enrichir à leurs dépens. La common decency réfère donc à une vertu à la portée des gens ordinaires. Elle ne me semble pas sans rapport avec les codes d'honneur

13. François Flahault, Pourquoi limiter l'expansion du capitalisme, Descartes \& Cie, Paris, 2003.

14 Dany-Robert Dufour, «La condition subjective dans les sociétés démocratiques » in L'anthropologie psychanalytique, sous la direction de Paul-Laurent Assoun et Markos Zafiropoulos, Anthropos, Paris, 2002, repris dans L'art de réduire les têtes..., op. cit.

15 Jean-Claude Michéa, Impasse Adam Smith, Climats, Castelnau-le-Lez, 2002. 
en vigueur dans les sociétés traditionnelles et surtout avec une sorte de conscience morale kantienne qui aurait été intuitivement mise en jeu dans les communautés organiques.

Or, la logique de l'échange marchand ne peut que toucher au cœur cette common decency dans la mesure où l'économie capitaliste n'admet aucune limitation, fut-elle celle de l'indécence. Bien au contraire même, l'indécence est devenue, dans l'économie capitaliste néo-libérale, la plus haute des vertus. C'est en effet une économie réglée sur une seule idée, celle de la richesse ou la puissance infinies. Ce que Anselm Jappe écrit fort bien:

elle [la société marchande] obéit à l'impulsion de s'accroître à tout prix, de transformer une somme d'argent en une somme toujours plus grande (...). Ce processus ne contient aucune limite naturelle ou sociale qui soit capable de constituer un point d'arrêt ${ }^{16}$.

Devant cette logique «folle» (le mot est de Marx, dans les Grundrisse, Tome I, p. 209), la common decency ne pèse évidemment plus rien.

En fait de socialité, l'échange marchand ayant tout consommé - le politique, l'économie des personnes et la common decency - ne propose plus qu'un principe organisateur, celui du réseau. D'où la vogue actuelle du réseau comme modèle même du lien social. Aujourd'hui, tout doit se mettre en réseau sous peine de pas être - les marchands et les marchandises, les informations, les artistes, les usagers de tel ou tel service, les malades (jusqu'aux schizophrènes et aux autistes), les associations émergentes, les groupes de pression, les universitaires et même les sociologues...

Or, le principe du réseau, c'est sa pure horizontalité. C'est Pierre Lévy qui me semble avoir le mieux résumé la logique du réseau, en intégrant la fonctionnalité technique spécifique du réseau informatique à la logique philosophique du rhizome de Deleuze ${ }^{17}$. Dans le réseau-rhizome, tout se passe en temps réel et en positivités. Rien ne manque, il suffit seulement pour un individu normalement pourvu de machines productives et/ou désirantes d'en brancher certaines dans le réseau pour que le «miracle» se produise, c'est-à-dire que «ça marche », c'est-àdire pour que le nouveau branché se trouve tout à coup comme au centre du système ${ }^{18}$. Les principes du réseau, fort simples, mais profondément subversifs dans leur utilitarisme et leur immanentisme mêmes, sont énonçables en quatre points ${ }^{19}$ :

- le principe de multiplicité signifie que le réseau est organisé sur un mode fractal; n'importe quel lieu peut lui-même se révéler composé de tout un réseau et ainsi de suite.

16 Anselm Jappe, Les aventures de la marchandise, op. cit., p. 70.

17 «Un rhizome ne commence et n'aboutit pas, il est toujours au milieu, entre les choses, inter-être (...). Le rhizome est alliance, uniquement alliance». On trouvera un véritable traité du «rhizome» dans Gilles Deleuze et Félix Guattari, Mille Plateaux, capitalisme et schizophrénie, Minuit, Paris, 1980, cf. «Introduction: rhizome», pp. 9-37.

18 La publicité joue beaucoup sur ce «miracle» opéré par le réseau: «dans cette banque, dans ce supermarché, vous êtes au centre du système...». On se souvient aussi que la Loi d'orientation de 1989 voulait mettre l'élève «au centre » du système scolaire...

19 Je m'inspire librement des travaux de Pierre Lévy, dont Les technologies de l'intelligence, La Découverte, Paris, 1990. 
- le principe d'extériorité spécifie que le réseau ne possède pas d'unité organique, son extension, sa diminution et sa recomposition peuvent toujours dépendre d'un branchement à d'autres réseaux.

- le principe topologique indique que, dans un réseau, il n'y a pas d'espace universel homogène où les messages ou informations ou marchandises circulent; ils créent l'espace dans lequel ils circulent, si bien que le réseau n'est pas dans l'espace, mais est l'espace.

- le principe de mobilité des centres énonce que le réseau possède en permanence plusieurs centres constamment mobiles.

On peut le constater: ce qui a tout simplement disparu dans le réseau-rhizome, c'est l'idée même de Loi tel qu'elle fonctionnait dans les ensembles symboliques, c'est-à-dire l'un en moins (le fameux «au-moins-un» de Lacan exposé, par exemple, dans L'Etourdit) qui permettait qu'un ensemble homogène se constitue. Tout, dans le réseau, se trouve au même plan, il n'existe que des interrelations mettant en rapports des acteurs. Il n'y a plus d'extériorité, que de l'intériorité. Plus de transcendance, que de l'immanence. D'où l'impression pour les «usagers» d'être toujours à l'intérieur, «au centre» du système. Le principe tiers a cédé la place à la relation duelle. Plus aucun acteur n'a de comptes à rendre à un tiers, à la fois très lointain et infiniment proche (présent en quiconque par exemple sous la forme du surmoi, de la loi morale, de la dette ou de la common decency), mais chacun est pris dans un ensemble de relations purement duelles. La Loi ne relève donc plus de catégories transcendantes, pas plus que de catégories symboliques (économie des personnes) ou de catégories communautaires (common decency), elle ne plus être recherchée que dans l'immanence des relations. Ce qui, bien sûr, ne peut que dépolitiser l'ensemble tout en multipliant les conflits. Lorsqu'un conflit entre deux acteurs survient, on n'en appelle pas à une loi (universelle, rendue au nom du Tiers), mais à une procédure (toujours locale) qui permet de remettre le circuit en marche.

C'est ainsi que le Marché, en tant que réseau, fût-il étendu aux limites du monde comme dans la globalisation actuelle, ne fait aucune place ni au manque, ni à un au-delà du sens. L'acteur est celui qui peut tout brancher dans le réseau, sauf ce qui peut éventuellement lui tenir le plus à cœur: un «pourquoi tout cela?», voire un «pour quoi et comment vivre?». Comme le disaient Deleuze et Guattari, qui semblaient s'en réjouir, dans le rhizome, «où allez-vous? d'où partez-vous? où voulez-vous en venir? sont des questions [devenues] bien inutiles». Car il s'agit dans cet univers de ne plus «commencer, ni finir $»^{20}$. Cette posture a, en tout cas, le mérite de la clarté: le réseau-rhizome nous prive des questions de l'origine et de la fin!

Certes, j'en conviens, ce sont là des interrogations absolument inutiles. Mais il ne semble pas qu'à éviter de les poser, on s'en porte beaucoup mieux. On pourrait d'ailleurs, au passage, se demander pourquoi des philosophes en sont venus à vouloir priver l'homme de ses «vaines » questions alors même qu'il n'y avait souvent qu'eux pour y faire droit en excluant délibérément de tout utilitarisme. Je

20 Gilles Deleuze et Félix Guattari, Mille Plateaux, capitalisme et schizophrénie, op. cit., p. 36. 
crois en somme que le consentement inconditionnel au réseau-rhizome est susceptible de rendre un fort mauvais service à l'homme en le privant explicitement de ces choses inutiles qui ne cessent cependant de l'intéresser, voire de le tourmenter. Par exemple, lorsqu'il parie sur l'au-delà de soi contre l'affirmation du moi et ses choix. Sur le définitivement impossible contre l'indéfiniment possible. Sur un pur absolu contre le relativisme généralisé du réseau. Sur le poème contre l'information $^{21}$. Sur ce qui se donne dans une phrase inouïe ou dans un geste héroïque contre toute forme d'utilités. Dessaisir l'homme de l'inutile ne représente-t-il pas le plus sûr moyen d'en faire un être en déshérence?

On peut d'ailleurs mettre l'extension du modèle du réseau en rapport avec les nouvelles formes prises par les troubles mentaux dans nos sociétés ${ }^{22}$. Comme le réseau ignore le Tiers et ne peut proposer que des relations duelles, c'est-à-dire des interactions, il ne permet pas au sujet de s'ombiliquer à ce qui le dépasse. Or, un sujet privé des questions impossibles de l'origine et de la fin, c'est un sujet amputé de l'ouverture à l'être, autrement dit un sujet empêché d'être pleinement sujet.

Le réseau constitue donc une sorte de degré zéro de la socialité puisqu'il forclôt tout rapport à l'être. C'est pourtant ce type de relation qui est aujourd'hui proposé comme le modèle de toute société possible. Nous sommes en effet tous devenus en puissance des «usagers» de réseaux. Or, le réseau ne peut que confronter chacun à la question de sa propre fondation, le laissant absolument seul face à une subjectivation qu'il se trouve contraint d'assumer par lui-même sans pouvoir nécessairement le faire. C'est tout le fonctionnement trinitaire de la condition subjective et du lien social dont j'essaie essayé de rendre compte dans Les mystères de la trinité $e^{23}$ qui se trouve ainsi mis en péril en produisant ses effets dévastateurs sur le sujet parlant. Le modèle du réseau nous fait passer d'un régime où l'inconscient se manifestait de façon prévalente par la névrose (comme dette à l'égard du tiers) à un mode où il se manifeste par des formes psychotisantes ${ }^{24}$. Il ne faudrait cependant pas croire que nos connaissances sur la bonne vieille psychose classique (paranoïaque ou schizophrénique) sauraient suffire à rendre compte du phénomène. Nous n'en sommes au contraire qu'au tout début de l'exploration des nouvelles formes psychotisantes de la post-modernitée ${ }^{25}$.

21 L'écrivain Pierre Michon semble exactement répondre à la condamnation de Deleuze et Guattari quant aux questions du début et de la fin lorsqu'il écrit que «les poèmes (...) peuvent servir à ça, tenir dans le même coup d'œil le Big Bang et le Jugement dernier (...). À quoi bon des poètes, en nos temps qui sont des temps de détresse? (...) Pour ça seulement ». Pierre Michon, Corps du roi, Verdier, Paris, 2002, pp. 74-75.

${ }^{22}$ L'étude de Alain Ehrenberg, La fatigue d'être soi (Odile Jacob, Paris, 1998) est à cet égard fort intéressante.

23 Dany-Robert Dufour, Les mystères de la trinité, Gallimard, Paris, 1990.

${ }^{24}$ Je me permets de renvoyer sur ce point à Dany-Robert Dufour, Folie et démocratie, Gallimard, Paris, 1996.

25 Je rejoins donc les hypothèses du psychanalyste Jean-Pierre Lebrun qui avance le terme de «sujet en état d'expérience limite» pour parler des «modalités cliniques nouvelles» dans lesquelles apparaît le «sujet d'aujourd'hui»: un sujet «laissé dans l'incapacité d'assumer par lui-même une subjectivation ». Jean-Pierre Lebrun, Les désarrois nouveaux du sujet, Érès, Paris, 2001, p. 66. 
Le processus de mise en réseau du monde est peut-être irrésistible. Mais, si puissant soit-il, le réseau ne peut qu'échouer - sur un point au moins, mais capital - à fonctionner comme fondateur de socialité. Loin de prendre en charge la question de l'origine, du fondement, de l'élément premier, c'est-à-dire la question très hégélienne du désir d'infini en l'homme, il ne peut que confronter chaque individu aux affres (qui ne vont certainement pas sans nouvelles jouissances) de l'auto-fondation. C'est là, sans doute, où se repère la limite fondamentale de l'économie de marché dans sa prétention à prendre en charge l'ensemble du lien personnel et du lien social: ce n'est pas une économie générale, pas une économie symbolique, mais seulement une «économie économique». Elle joue certes dans le registre libidinal, dans la mesure où elle prétend toujours présenter à tout sujet un objet manufacturé supposé venir combler son désir, mais elle échoue à fonctionner comme économie générale dans la mesure où elle laisse le sujet face à luimême pour l'essentiel: sa propre fondation. Or, si cette (impossible) question de l'origine n'est pas traitée, elle ne peut revenir que comme irrépressible tourment. Il s'agit là en effet d'une question qui ne peut être abrogée, mais seulement élaborée dans et par la culture, dans ce que Freud appelait un Kulturarbeit qu'il présentait comme «un travail interminable, à reprendre sans cesse et sans relâche» pour que «je» advienne ${ }^{26}$. Ce travail spécifique de la culture, nécessaire à l'avènement du «je», ne pouvant être accompli par le Marché et ses réseaux d'échanges, ce sont fréquemment les revendications identitaires les plus folles qui se présentent alors (fondamentalismes, ethnicismes, régionalismes, individualismes...).

\section{POUR UNE AUTRE SCIENCE SOCIALE}

Pour conclure, il me semble que les sciences sociales contemporaines se sont, sauf notables exceptions, plutôt laissées prendre au piège de l'économie de marché. Il est remarquable au demeurant que les postures «contestataires» n'échappent pas à ce diagnostic - bien au contraire comme tend à le prouver l'étude de Boltanski et Chiapello sur l'intégration de la «critique artistique» dans le «nouvel esprit du capitalisme $»^{27}$. De façon générale, les sciences sociales ont voulu continuer à trouver du social là où il n'existait quasiment plus parce que désormais entièrement subordonné à des rapports économiques marchands. Elles sont même allées très loin dans ce sens, allant jusqu'à fouiller de façon acharnée les lieux les plus ténus (on fait la sociologie des rollers, des hackers, des obèses, des transsexuels, de tous les aspects de la vie quotidienne et même la sociologie du sujet). Certes, tant qu'il y aura des sociologues, il faudra bien qu'ils trouvent des objets. Mais plus ils en trouvent, plus le social lui-même semble introuvable. Cette recherche, qui a quelque chose de pathétique, se manifeste dans l'extrême diversification des objets et des approches de la sociologie actuelle. On dispose ainsi de quantités de micro-sociologies qui examinent la position de chaque

\footnotetext{
26 Freud, Nouvelles conférences sur la psychanalyse [1932], Paris, Gallimard, 1989, p. 110, où se rencontre la fameuse formule: «Wo Es war, soll Ich werden. Es ist Kulturarbeit etwa wie die Trockenlegung der Zuydersee».

27 Luc Boltanski et Eve Chiapello, Le Nouvel esprit du capitalisme, Gallimard, Paris, 1999.
} 
acteur. On dispose d'études qui cherchent à établir, de façon relativiste, les normes et valeurs de chaque groupe comme si chacun était isolable de l'ensemble. On dispose enfin d'approches subjectivistes où tout rapport à l'autre, constitutif de la posture de recherche, a disparu. On confond alors allègrement le fait que l'autre parle en moi avec le fait qu'il parle $d e$ moi $^{28}$. Dans ce positionnement, très répandu (cf. les théories de l'implication), les sciences sociales tendent à se nier ellesmêmes puisque le récit tend alors à se substituer à la recherche. Si, au moins, celui-ci avait une tenue littéraire minimale, nous n'aurions affaire qu'à un demimal. Mais le cas est plutôt rare.

Il ne me semble pas qu'une science sociale puisse exister à nouveau sans qu'elle prenne pour objet l'ensemble. Il est vrai que la tâche est énorme parce qu'il faudra intégrer en un tout cohérent des données venant de l'économie marchande, de l'économie politique, de l'économie symbolique et l'économie psychique. Mais, pour être démesurée, la tâche n'en est pas moins indispensable, voire même urgente. Il me semble en effet qu'un nouvel être social est en formation. Un homme déchu de sa faculté de juger, poussé à jouir sans désirer, délié de la dette symbolique, revendiquant l'indécence comme nouvelle vertu est en effet en train d'apparaître sous nos yeux et s'imposer comme nouvelle norme. Il serait dommage que la sociologie, perdue dans ses micro-approches, n'ait rien vu venir.

UFR Arts, Philosophie et Esthétique

Département des sciences de l'éducation

Université de Vincennes, Paris VIII

Sur le rapport à l'autre, constitutif de tout dispositif de recherche, et sur la critique des approches subjectivistes, voir Marilia Amorim, Dialogisme et altérité dans les sciences humaines, L'Harmattan, Paris, 1996. 